\title{
Energy landscape - a key concept in the dynamics of liquids and glasses
}

\author{
U. Buchenau \& \\ Institut für Festkörperforschung, Forschungszentrum Jülich, Postfach 1913, 52425 \\ Jülich, Germany
}

\begin{abstract}
There is a growing belief that the mode coupling theory is the proper microscopic theory for the dynamics of the undercooled liquid above and around a critical temperature $T_{c}$. In addition, there is some evidence that the system leaves the saddlepoints of the energy landscape to settle in the valleys at this critical temperature. Finally, there is a microscopic theory for the entropy well below $T_{c}$ (i.e. close to the calorimetric glass transition $T_{g}$ ) by Mézard and Parisi, which allows to calculate the Kauzmann temperature from the atomic pair potentials.

The dynamics of the frozen glass phase is at present limited to phenomenological models. In the spirit of the energy landscape concept, one considers an ensemble of independent asymmetric double-well potentials with a wide distribution of barrier heights and asymmetries (ADWP or Gilroy-Phillips model). The model gives an excellent description of the relaxation of glasses up to about $T_{g} / 4$. Above this temperature, the interaction between different relaxation centers begins to play a role. In a mean-field treatment, the interaction reduces the number of relaxation centers needed to bring the shear modulus down to zero by a factor of three.

PACS numbers: 64.70.Pf, 62.40.+i
\end{abstract}

$\S$ u.buchenau@fz-juelich.de 


\section{Introduction}

In the authors view, our present foggy picture of the glass transition begins to show some cornerstones of a future solid theoretical building, namely the energy landscape concept (Goldstein 1968; Johari and Goldstein 1970, 1971; Stillinger 1995), the mode coupling theory (Bengtzelius et al 1984; Götze and Sjögren 1992) together with the realization (Bengtzelius et al 1984; Angell 1988) that the critical temperature of this theory marks the onset of thermally activated motion between the minima of the energy landscape, and, finally, the calculation of the Kauzmann temperature from the interatomic potentials (Mézard and Parisi 1996, 1999). These theories explain, at least in principle, the dynamics above and around $T_{c}$ as well as the thermodynamics near to the calorimetric glass transition $T_{g}$, leaving only the fragility (Angell 1988; Böhmer et al 1993; Angell 1995) without a solid theoretical foundation.

The present paper expands this view in a bit more detail in the next two sections. Section 4 addresses the relaxation in glasses at temperatures well below $T_{g}$ in terms of thermally activated jumps in an ensemble of independent asymmetric double-well potentials (the ADWP (ㅅsymmetric Double Well Potential) (Pollak and Pike 1972) or Gilroy-Phillips model (Gilroy and Phillips 1981)). Section 5 considers the effect of the interaction between different relaxation centers, which is shown to become dominant at the glass transition. Section 6 compares the prediction of a mean field treatment to experimental data on the breakdown of the shear modulus. Conclusions are given in Section 7.

\section{2. $T_{c}$ : The onset of thermally activated motion}

The mode coupling theory of the glass transition (Götze and Sjögren 1992) does not require the concept of the energy landscape. In fact, its most impressive experimental proof was found in colloids (Pusey and van Megen 1986; van Megen and Underwood 1994), which do not have an energy landscape.

On the other hand, the dynamics of the undercooled liquid at lower temperatures is dominated by thermally activated hopping between different valleys of the energy landscape. The conjecture (Bengtzelius et al 1984; Angell 1988) of an onset of this thermally activated motion at the critical temperature $T_{c}$ of the mode coupling theory has found more and more support from numerical simulations of model glass formers (Schrøder et al 2000; Angelani et al 2000; Broderix et al 2000; but see also Doliwa and Heuer 2002, which stress the important role of thermal activation above $T_{c}$ ). The main result is illustrated in Fig. 1, adapted from Angelani et al (2000). As one lowers the temperature towards $T_{c}$, the average number of saddle points of the energy landscape on which the system finds itself at a given moment in time decreases. This number extrapolates to zero at $T_{c}$. At $T_{c}$, one still finds a finite number of unstable instantaneous normal modes. However, these stem from shoulders of the potential with a negative curvature rather than from true saddle points. They give rise to the fast picosecond 
motion (Angell 1995), but they do not dominate the long time dynamics.

Above $T_{c}$, the separation of the $\alpha$-process (the elementary process of the flow) from the microscopic picosecond motion (Franosch et al 1998) in an undercooled liquid seems to be reasonably well described by the mode coupling theory. This was demonstrated by neutron (Knaak et al 1988; Frick et al 1991; Wuttke et al 1993) and light scattering experiments (Li et al 1992; Sokolov 1998; Wiedersich et al 2000a) on ionic, molecular and polymeric glass formers, as well as in a number of numerical simulations (Kob and Andersen 1995a, 1995b; Nauroth and Kob 1997; Kammerer et al 1998a, 1998b). There are more examples (Götze 1999). One finds the proper scaling relations for the time and temperature dependence of the $\alpha$-process and the fast picosecond $\beta$ process, consistent with the exponents determined from the temperature dependence of the viscosity above $T_{c}$. As a general rule (Sokolov 1998), one finds $\tau_{\alpha}\left(T_{c}\right) \approx 10^{-7} \mathrm{~s}$, a bit longer than the originally considered value (Goldstein 1968; Angell 1988) of $10^{-9} \mathrm{~s}$.

Note this does not imply a perfect fit of theory and experimental data. Though one observes the expected power law behaviour of the viscosity in many liquids (Taborek et al 1986), accurate dielectric data in salol show a temperature dependence of the power law exponent of $\tau_{\alpha}$ even well above $T_{c}$ (Stickel et al 1995). Quantitative checks of the theory are at present impeded by the difficulty to calculate $T_{c}$ and the exponents for most real glass formers.

Nevertheless, the mode coupling theory is a true microscopic theory, which in

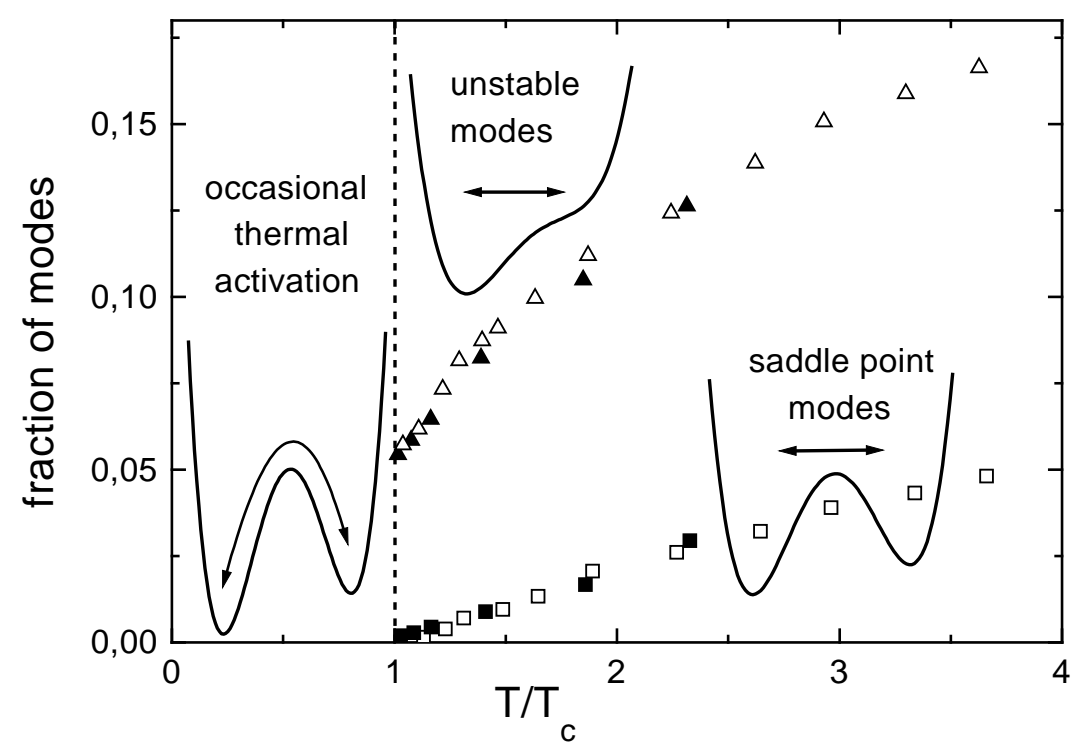

Figure 1. Temperature dependence of the fraction of saddle-point modes (squares) and of instantaneous unstable normal modes (triangles) in two Lennard-Jones glass formers after Angelani et al (2000). 
principle allows to calculate its parameters from a knowledge of the interatomic potentials. The results obtained so far seem to show that one can identify the critical temperature of this microscopic theory with the temperature at which the system leaves the saddle points of the energy landscape to settle in its valleys. Below $T_{c}$, one expects a gradual transition to thermally activated energy landscape dynamics. Though there is no generally agreed description of this landscape dynamics, there is at least a microscopic theory (Mézard and Parisi 1996, 1999) for the thermodynamics of the undercooled liquid at this lower temperature.

\section{Calculating the Kauzmann temperature}

This further important theoretical progress of the last decade concerns an old concept from the thermodynamics of the glass transition, the Kauzmann temperature. The concept stems from the experimental observation that the entropy difference between undercooled liquid and crystal seems to extrapolate to zero at a finite temperature, the Kauzmann temperature $T_{K}$, at which the glass former in principle condenses into a single structural configuration. Since the viscosity depends on the number of accessible configurations, one expects a divergence of the viscosity at about the same temperature (an excellent review of the older empirical attempts to model the thermodynamics and kinetics of the undercooled liquid has been given by Jäckle (1986)). In this general sense, the Kauzmann temperature is not only important for the thermodynamics, but also for the dynamics close to $T_{g}$. In fact, the empirical Adam-Gibbs model identifies the temperature $T_{K}$ with the Vogel-Fulcher temperature $T_{0}$ of the empirical VFT (VogelFulcher-Tammann) or WLF (Williams-Landel-Ferry (Ferry 1980)) relation

$$
\tau_{\alpha}=\tau_{0} \mathrm{e}^{A /\left(T-T_{0}\right)},
$$

where $\tau_{0}$ is a microscopic time and $A$ is a second parameter of this empirical relation. If one looks more closely (Stickel et al 1995, 1996; Hansen et al 1997), the VogelFulcher relation does not describe the temperature dependence of $\tau_{\alpha}$ very well. From this data collection, one rather feels that each glass former behaves differently below $T_{c}$. Nevertheless, the general tendency of a divergence of the viscosity as the glass former looses its configurational entropy cannot be denied.

Mézard and Parisi (1996, 1999) have devised a recipe to calculate the entropy and the Kauzmann temperature from the pair potentials of a given glass former, thus providing the Kauzmann extrapolation scheme with a theoretical solidity which it lacked before. The calculation assumes an undercooled liquid below $T_{c}$ which spends most of its time vibrating in a local minimum of the free energy, with only occasional jumps into a neighboring minimum. In this situation, one assumes the validity of the harmonic approximation for the motion within the single well. Using the replica concept, one calculates the free energy as a function of temperature and finds a nonzero Kauzmann temperature. This is again a microscopic theory, because it allows to calculate the heat capacity and the Kauzmann temperature from the pair potentials between the atoms. 
Though the Kauzmann temperature itself might still prove to be an artefact of the mean-field approximation of the calculation, the calculated heat capacity above this temperature resembles measured data above $T_{g}$ both in shape and size.

The next section proceeds to a phenomenological model of the thermally activated energy landscape dynamics in the low-temperature glass phase.

\section{ADWP or Gilroy-Phillips model}

The ADWP (Asymmetric-Double-Well-Potential) (Pollak and Pike 1972) or GilroyPhillips model (Gilroy and Phillips 1981) is a member of a family of three glass models, which are essentially one and the same model applied to three different situations (the other two are the tunneling model (Phillips 1981) and the soft-potential model (Parshin 1994)). The basic idea is to simplify the multiminimum situation of the energy landscape to an ensemble of independent double-well potentials for local structural rearrangements with a broad distribution of different barrier heights $V$ and asymmetries $\Delta$ between the two minima (see Fig. 2) - an inherently heterogeneous description of the dynamics of the glass phase (for a review on the heterogeneity of undercooled liquids see Richert 2002).

Consider a single relaxing entity, i.e. a single barrier of height $V$ separating two neighboring energy minima. Fig. 2 shows schematically the energy as a function of the configurational coordinate going from one minimum to the other. In numerical simulations of model glasses, one finds that this configurational coordinate involves the motion of about five to fifty atoms in the center of the relaxing entity (Heuer and Silbey 1996; Schober and Oligschleger 1996). There is no reason why the two minima should have the same energy, so there will be an energy difference $\Delta$ between them. A further characteristic of the two adjacent minima is the coupling of this relaxing entity to the external shear strain $\epsilon$. This is given by the coupling constant $\gamma$, defined such that the asymmetry changes from $\Delta$ to $\Delta+\gamma \epsilon$ under the applied shear strain $\epsilon$.

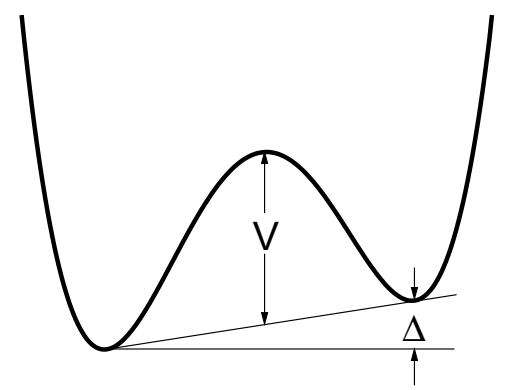

Figure 2. Asymmetric double-well potential. 
With these three energies, the barrier height $V$, the asymmetry $\Delta$ and the coupling constant $\gamma$, one can quantify the contribution of this single relaxing entity to the dynamical mechanical behaviour of the viscoelastic medium. The relaxation time $\tau_{V}$ is given by the Arrhenius relation

$$
\tau_{V}=\tau_{0} \mathrm{e}^{V / k_{B} T}
$$

where $\tau_{0}$ is a microscopic time of the order of $10^{-13}$ seconds, $V$ is the energy of the barrier between two energy minima of the system, and $T$ is the temperature.

In the simplest possible approximation, the free energy $F$ of the single relaxation center reads

$$
F=-k_{B} T \ln \left[2 \cosh \left(\frac{\Delta+\gamma \epsilon}{2 k_{B} T}\right)\right] .
$$

Its second derivative with respect to the shear distortion $\epsilon$ is

$$
\frac{\partial^{2} F}{\partial \epsilon^{2}}=-\frac{\gamma^{2}}{4 k_{B} T \cosh ^{2}\left(\Delta / 2 k_{B} T\right)}
$$

The second derivative determines the contribution of the specific relaxing entity to the difference between the shear moduli at infinite and zero frequency. The main influence on the shear modulus is due to relaxation in potentials with asymmetries smaller than $k_{B} T$; for larger asymmetries the influence decreases rapidly because of the square of the hyperbolic cosine in the denominator.

We assume a number density function $n(V, \Delta)$ of these local structural relaxations, which varies little if $V$ or $\Delta$ change by energies of the order $k_{B} T$. Integrating over the asymmetry (Buchenau 2001), we obtain a net difference $\delta G$ between the infinite and the zero frequency shear moduli due to relaxations with barrier heights between $V$ and $V+\delta V$

$$
\frac{\delta G}{G \delta V}=\frac{\gamma^{2} n(V, 0)}{G} \equiv f_{0}(V)
$$

where $G$ is the infinite frequency shear modulus. This relation defines $f_{0}(V)$, the barrier density function without interaction.

In the frozen glass phase, one expects a frozen-in distribution of barrier heights and asymmetries and thus a temperature-independent $f_{0}(V)$. One can check that by looking at the distribution with low and high frequencies; according to the Arrhenius relation, eq. (2), one should be able to observe the same barrier height at different temperatures. Such checks have been done for a number of different glass formers. As long as one stays at temperatures much lower than the glass temperature, one finds impressive agreement with the idea of a temperature-independent barrier density function. One example is vitreous silica up to $300 \mathrm{~K}$, glass temperature $1473 \mathrm{~K}$ (Wiedersich et al 2000b). Another one is polymethylmethacrylate (PMMA) below $80 \mathrm{~K}$, glass temperature $383 \mathrm{~K}$ (Buchenau et al 2002). For higher temperatures, however, the barrier density function tends to increase with increasing temperature (Surovtsev et al 1998, Caliskan et al 2002). 
As will be shown in the next section, one has to expect such an increase, because the interaction between different relaxation centers determines the dynamics close to the breakdown of the shear modulus. In this view, the Johari-Goldstein $\beta$-process, a broad relaxation maximum close to the $\alpha$-process which shows an Arrhenius behaviour both below and above $T_{g}$ (Johari and Goldstein 1970, 1971; Kudlik et al 1999) corresponds to a peak in $f_{0}(V)$, strongly enhanced by the proximity of the breakdown.

\section{The 1/3-rule}

For the purpose of this and the following section, let us assume that the decomposition of the complex energy landscape into an ensemble of single relaxation centers or single relaxing entities is a reasonable and solid basis. In a mean-field scheme, the interaction between the single entity and all the others is taken into account by embedding the single center into the viscoelastic medium, calculating the viscoelastic properties selfconsistently.

The first implication of this mean-field assumption is that the asymmetry $\Delta$ is no longer fixed, but changes on the Maxwell time scale, because the viscoelastic medium is free to flow. Consequently, the term $\gamma \epsilon$ in eq. (3) can adapt on the Maxwell time scale, thus changing $\Delta$ to a different value. The Maxwell time $\tau_{M}$ is given by the shear viscosity $\eta$ and the infinite frequency shear modulus $G$

$$
\tau_{M}=\eta / G,
$$

where all three quantities depend on temperature, the infinite frequency shear modulus $G$ only weakly, but the two other quantities drastically.

A change in $\Delta$ does not mean that the energy landscape itself flows; to take a threedimensional example, the barrier is like a ridge between two sloping valleys with different slope; going along the ridge changes the height difference of the two valleys. In this example, the coordinate along the ridge could correspond to the external shear strain; then one has a continuous change of $\Delta$. It could also correspond to the configurational coordinate of another relaxing entity in the neighborhood, which changes the local shear strain at the given relaxation center in a discontinuous way.

It is interesting to consider the consequences of a freely changing value of $\Delta$. Taking eq. (3) literally, one calculates a Boltzmann factor of the relaxing entity

$$
\exp \left(-F / k_{B} T\right)=2 \cosh \left(\Delta / 2 k_{B} T\right),
$$

which has its lowest value at the symmetric case, $\Delta=0$, and diverges with increasing $\Delta$. This latter feature is of course unphysical, because one cannot expect to gain energy without limit by increasing the asymmetry of such a local entity. However, the consideration shows that one must expect a relatively low probability for the symmetric

case, because it is energetically unfavorable. In fact, both the number of tunneling states and the excess entropy of the frozen glass seem to decrease in selenium upon aging (Johari 1986). 
As soon as $\Delta$ is able to change, the relaxing entity has an additional possibility to find its thermal equilibrium, namely by lowering the energy of the minimum in which the system happens to find itself. It is therefore natural to assume that relaxation centers with high barriers, whose relaxation time exceeds the Maxwell time, do not contribute to the viscoelastic properties of the medium, while those with shorter relaxation times have time to equilibrate by jumps over the barrier and do contribute. The two barrier regimes are separated by the Maxwell barrier $V_{M}$ with

$$
V_{M}=k_{B} T \ln \left(\tau_{M} / \tau_{0}\right) .
$$

Consider the energetics of a single double-well, with a barrier low enough to equilibrate within the Maxwell time. Suppose a small constant shear strain $\epsilon$ is switched on at time zero, with the population of the minima of the double-well in thermal equilibrium with respect to zero shear strain. The new thermal equilibrium requires a number of jumps

$$
\delta n=\frac{\gamma \epsilon}{4 k_{B} T \cosh ^{2}\left(\Delta / 2 k_{B} T\right)} .
$$

In order to calculate the energy $\delta U$ carried to the heat bath, we have to multiply the number of jumps $\delta n$ with the energy difference $\Delta+\gamma \epsilon$. Therefore these jumps transport the energy

$$
\delta U=\frac{\Delta \gamma \epsilon+\gamma^{2} \epsilon^{2}}{4 k_{B} T \cosh ^{2}\left(\Delta / 2 k_{B} T\right)}
$$

from the macroscopic shear stress energy to the heat bath. The term on the right hand side linear in $\epsilon$ must be compensated by other relaxing entities with opposite sign of $\Delta$ (otherwise there would be no initial equilibrium). If one compares the second quadratic term $\delta U_{2}$ with the second derivative of the free energy in eq. (4), one finds that it is twice as high as the free energy decrease $\delta F$ calculated from eq. (雨)

$$
\delta F=\frac{\gamma^{2} \epsilon^{2}}{8 k_{B} T \cosh ^{2}\left(\Delta / 2 k_{B} T\right)}=\frac{1}{2} \delta U_{2},
$$

which determines the reduction of the shear modulus by the barrier. The physical reason for this is the reduced entropy; spending the energy one has spanned an entropic spring. Thus the reduction of the shear modulus is only half that expected from the spent energy. This is in principle textbook knowledge for a Debye relaxation, but is explained here again, because it is essential for the understanding of the glass transition.

In a Gedankenexperiment, let the thermally equilibrated relaxation center return from the actual asymmetry $\Delta+\gamma \epsilon$ in the strained state to its original asymmetry $\Delta$, say by appropriate jumps in the surroundings which change the local strain at the center. In principle, this return would again require the energy $\delta U$, to be taken again from the macroscopic stress energy. However, this return occurs on the Maxwell time scale, which is long compared to the relaxation time of the relaxation center. Therefore the population of its two minima adapts adiabatically. The number of backjumps is again the same $\delta n$, but now the asymmetry reduces gradually from $\Delta+\gamma \epsilon$ to $\Delta$ in the course 
of the process, reducing the average energy per jump to $\Delta+\gamma \epsilon / 2$. This means one needs only the energy $\delta U_{2}-\delta F=\delta F$ to return.

Now imagine this happens for all relaxation centers with barriers lower than the Maxwell barrier of eq. (8) in the undercooled liquid. Then one returns to an unstrained equilibrium in the strained state. This means a full relaxation of the initial stress, as in the true flow process characterized by the Maxwell time. Naturally, our Gedankenexperiment is a rather improbable realization of this process, because in the real process a given relaxation center will almost never return to its initial asymmetry, though the macroscopic stress relaxes back to zero. However, this special realization allows to keep track of the energy contributions.

In this cycle from the initial equilibrium to a new equilibrium in the strained state, each barrier with $\tau_{V}$ smaller than $\tau_{M}$ takes the energy $3 \delta F$ from the stress energy, $2 \delta F$ in the initial equilibration and $\delta F$ on the Maxwell time scale. The direct reduction of the shear modulus, the one expected if there were no interaction between different barriers, corresponds only to a single $\delta F$. The energy $3 \delta F$ is taken from the potential energy in the strain field, reducing it to zero. One arrives at the conclusion that the stress relaxation occurs when the noninteracting relaxation centers reduce the shear modulus by one third of its infinite frequency value. To put it differently, the interaction between relaxing entities reduces the number needed to bring the long time shear modulus down to zero by a factor of three.

In terms of the barrier density function $f_{0}(V)$, this means

$$
\int_{0}^{V_{M}} f_{0}(V) d V=\frac{1}{3} .
$$

This is the 1/3-rule, which allows to calculate the Maxwell barrier (and from the Maxwell barrier, the shear viscosity) for a given barrier density function $f_{0}(V)$. The barrier density function $f_{0}(V)$ in turn can be determined from measurements at times shorter than the Maxwell time. To do this, one needs a quantitative treatment of the interaction between different relaxation centers, which is the topic of the next section.

The 1/3-rule provides a qualitative understanding of the fragility: As the temperature increases, $f_{0}(V)$ is expected to increase, because symmetric double-well potentials are energetically unfavorable, as pointed out at the beginning of this section. This implies that $V_{M}$ decreases with increasing temperature, as one indeed observes in experiment. The problem is to make this understanding quantitative. One could hope to use the theoretical tools of Mézard and Parisi $(1996,1999)$ to achieve this end.

\section{The breakdown of the shear modulus}

The 1/3-rule, eq. (12), can be derived independently (Buchenau 2002) from the assumption

$$
\frac{\delta G}{G \delta V}=f_{0}(V) \frac{G^{2}}{G\left(\tau_{V}\right)^{2}} \mathrm{e}^{-\tau_{V} / \tau_{M}} \equiv f(V) .
$$


Eq. (13) is a generalization of the definition of the barrier density function $f_{0}(V)$ without interaction, eq. (5), to describe the enhancement of the effect of a single relaxation center by the interaction, together with the cutoff at the Maxwell time. The equation defines a barrier density function $f(V)$ with interaction, which can then be used to calculate the full shear response.

One can justify the quadratic enhancement factor assuming a constant strain applied at time zero. The relaxation will tend to equilibrate at the time $\tau_{V}$, when the square of the stress - a measure of the remaining stress energy - is reduced by precisely this factor, while the number of jumps required for the equilibration may be taken to be unchanged. If the number of jumps and the distortion remain unchanged, the energy and the free energy contribution remain unchanged. This implies that the reduction of the stress energy by the relaxation is also unchanged. This in turn increases the apparent barrier density function $f(V)$ by the square of $G / G\left(\tau_{V}\right)$. A physical interpretation of this increase are lower-barrier jumps, taking place in the neighborhood after a jump of the central entity.

One can do a selfconsistent calculation of $f(V)$ for a given $f_{0}(V)$ by inserting the expression for $G\left(\tau_{V}\right)$ in terms of $f(V)$ into eq. (13). This leads to an integral equation for $f(V)$ with the approximate solution (Buchenau 2002)

$$
f(V)=\frac{f_{0}(V) \exp \left(-\tau_{V} / \tau_{M}\right)}{\left[3 \int_{0}^{\infty} \exp \left(-\tau_{V} / \tau_{v}\right) \exp \left(-\tau_{V} / \tau_{M}\right) f_{0}(v) d v\right]^{2 / 3}},
$$

where the Maxwell time is again given by the $1 / 3$-rule, eq. (12).

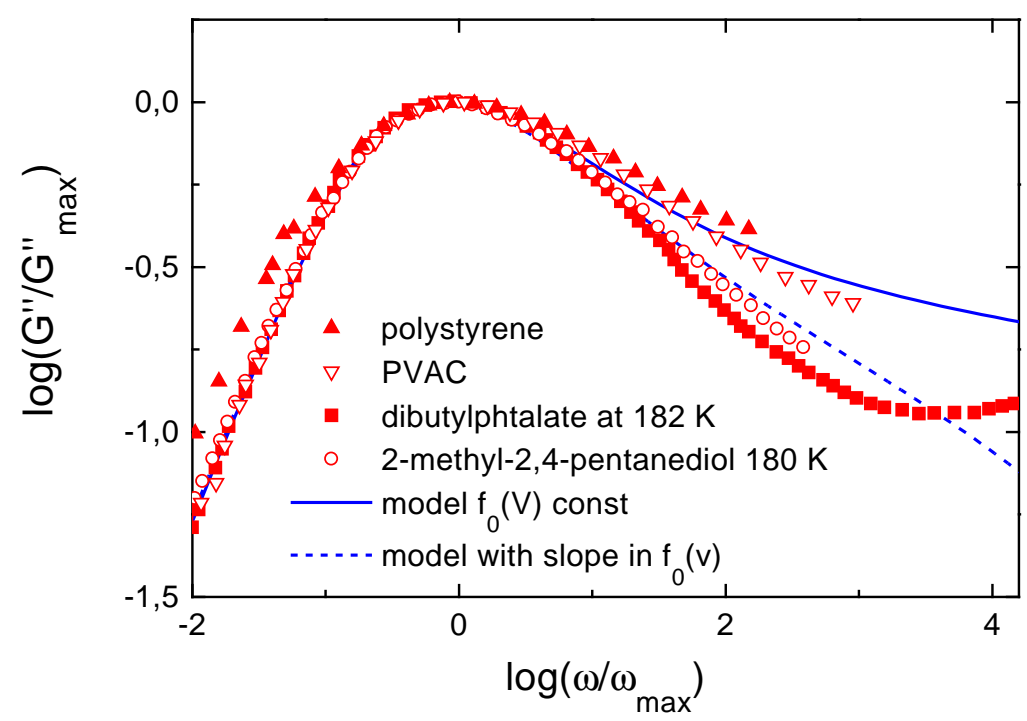

Figure 3. Shape of the normalized $\alpha$-peak in $G^{\prime \prime}(\omega)$ in a log-log plot for two polymers (Donth et al 1996) and the two molecular glass formers dibutylphtalate (Behrens et al 1996) and 2-methyl-2,4-pentanediol (Christensen and Olsen 1995). The continuous line shows the model, the dashed line the adapted model (see text). 
The denominator of eq. (14) tends toward zero as $V$ approaches the Maxwell barrier. Therefore the breakdown of the shear modulus occurs in a rather dramatic way. The relaxing entities at this critical barrier value receive a strong enhancement, to such an extent that one is tempted to assume a separate $\alpha$-process which has nothing to do with the secondary relaxations. In fact, this more or less unconscious assumption underlies most of the present attempts to understand the glass transition (Ediger et al 1996). The above mean-field treatment shows such an assumption to be unnecessary; what one sees at the glass transition are simple Arrhenius relaxations of no particularly large number density, blown up to impressive size by the small denominator of eq. (14).

Once $f(V)$ is known, one can determine the frequency dependence of the complex shear modulus at the frequency $\omega$ from the two relations

$$
G^{\prime}(\omega)=G \int_{0}^{\infty} f(V) \frac{\omega^{2} \tau_{V}^{2} d V}{1+\omega^{2} \tau_{V}^{2}}
$$

and

$$
G^{\prime \prime}(\omega)=G \int_{0}^{\infty} f(V) \frac{\omega \tau_{V} d V}{1+\omega^{2} \tau_{V}^{2}} .
$$

One must expect $f_{0}(V)$ and, consequently, $f(V)$ to be a different function for each glass former. In many of the cases, however, one should be able to approximate $f_{0}(V)$ by the constant value $f_{0}\left(V_{M}\right)$ for barriers close to $V_{M}$. Such a choice is also suggested by the appearance of a constant loss term (Kudlik et al 1999; Ngai 2000; Sokolov et al 2001) in many glass formers just below $T_{g}$. A constant loss term corresponds to a constant $f(V)$. In the model with a barrier-independent $f_{0}(V), f(V)$ decreases and approaches $f_{0}(V)$ as one goes from the Maxwell barrier to lower barriers. For this generic case, one can calculate $G^{\prime}$ and $G^{\prime \prime}$ at the breakdown of the shear modulus and compare the result to measured data.

There are some good mechanical shear measurements over many decades in frequency at $T_{g}$ (Christensen and Olsen 1994; Christensen and Olsen 1995; Behrens et al 1996; Donth et al 1996). Fig. 3 shows data for $G^{\prime \prime}(\omega)$, normalized to the peak maximum. The continuous line is calculated from the model assuming $f_{0}(V)=$ const and using eqs. (14) and (16). As can be seen from Fig. 3, the fit is only good for the two polymers polystyrene and poly(vinyl acetate); the two molecular glass formers show a much stronger decay of $G^{\prime \prime}$ towards the high frequency end. For these, the model gives a peak in the imaginary part of the shear modulus which is too small and too broad; the real breakdown of the shear modulus is more dramatic than the mean field calculation. A better fit requires an increase of $f_{0}(V)$ at the Maxwell barrier (the dashed line in Fig. 3). One can rationalize this increase; it might be necessary to reach a large peak in $f_{0}(V)$ before the shear modulus breaks down.

Dielectric measurements (Kudlik et al 1999) provide much more accurate peak shapes than mechanical ones. However, these data are usually presented as real and imaginary part of the dielectric constant, which is essentially a susceptibility. The model discussed here calculates moduli. These should be identical with the dielectric ones as long as the weighting of the relaxation centers according to the electric dipole moments 
corresponds to the one of the mechanical shear coupling constants. A comparison requires a conversion of the dielectric constants to dielectric moduli (Dyre 1991), which changes the peak shape considerably. Christensen and Olsen (1994) show three examples where dielectric moduli data give exactly the same peak shape as mechanical shear modulus data.

\section{Conclusions}

There begins to be a general agreement that the mode coupling theory of the glass transition (Götze and Sjögren 1992) is the proper microscopic theory for the separation of the structural relaxation time from the microscopic picosecond time scale in undercooled liquids. However, the structural relaxation time of real liquids does not diverge at the critical temperature $T_{c}$ of the theory. It rather reaches a a typical value between a nanosecond and a microsecond (Sokolov 1998), indicating a crossover to a different flow mechanism.

Numerical simulations (Schrøder et al 2000; Angelani et al 2000; Broderix et al 2000; but see also Doliwa and Heuer 2002) corroborate the old conjecture (Bengtzelius et al 1984; Angell 1988) that at $T_{c}$ the system leaves the saddle points of the energy landscape to settle in the valleys.

At still lower temperatures, one can use the approximation of harmonic energy minima, with only occasional thermally activated jumps in between. This is the starting point of a second microscopic theory (Mézard and Parisi 1996, 1999) for the entropy of the undercooled liquid, which allows to calculate the puzzling heat capacity at the calorimetric glass transition from the interatomic potentials. These two theories provide some microscopic insight (though one could wish for theories which are easier to handle).

The breakdown of the shear modulus at the calorimetric glass transition has as yet no microscopic explanation. One can show that it cannot be treated in terms of independent double-well relaxation centers (Pollak and Pike 1972; Gilroy and Phillips 1981), because the interaction becomes dominant at this breakdown, reducing the number of relaxation centers needed for the breakdown by a factor of three (the $1 / 3$ rule). A mean-field treatment (Buchenau 2002) of the interaction allows to calculate the shear response at the breakdown. The comparison to measured data shows agreement in some glass formers, but a more pronounced breakdown in others. One can rationalize this finding by postulating a rise of the barrier density function $f_{0}(V)$ at the Maxwell barrier for the latter ones.

A disappointing feature of the relaxation center picture is that one needs a whole temperature-dependent function, the barrier density function $f_{0}(V)$, to describe the dynamics around $T_{g}$. It is not enough to classify glass formers as type A or B (Kudlik et al 1999), depending on whether $f_{0}(V)$ shows a strong peak (Johari-Goldstein peak) below $V_{M}$ or not. On the other hand, this is a logical consequence of the validity of the energy landscape idea, because the energy landscape is different in different glass formers. If all relaxation centers up to the Maxwell barrier contribute to the flow process, 
then the description of its temperature and frequency dependence cannot be done by a single parameter. Below $T_{c}$, each glass former develops its own identity, a conclusion supported by experiment (Stickel et al 1995, 1996; Hansen et al 1997).

\section{Acknowledgments}

An enlightening talk on the current theories of the glass transition by R. Schilling at the Heraeus Summer School 2002 in Chemnitz was very helpful. Discussions with W.

Götze, A. Heuer, R. Richert and H. R. Schober are gratefully acknowledged.

\section{References}

Adam G and Gibbs J H 1965 J. Chem. Phys. 43139

Angelani L, Di Leonardo R, Ruocco G, Scala A and Sciortino F 2000 Phys. Rev. Lett. 855356

Angell C A 1988 J. Phys. Chem. Solids 49863

Angell C A 1995 Science $\mathbf{2 6 7} 1924$

Behrens C F, Christiansen T G, Christensen T, Dyre J C and Olsen N B Phys. Rev. Lett. 761553 (1996)

Bengtzelius U, Götze W and Sjölander A 1984 J. Phys. C 175915

Böhmer R, Ngai K L, Angell C A and Plazek D J 1993 J. Chem. Phys. 994201

Broderix K, Bhattacharya K K, Cavagna A, Zippelius A and Giardina I 2000 Phys. Rev. Lett. 855360

Buchenau U 2001 Phys. Rev. B 63104203

Buchenau U, Wischnewski A, Zorn R and Hadjichristides N 2002 Phil. Mag. B 82209

Buchenau U 2002 A mean-field model of the glass transition preprint cond-mat/0202036

Caliskan G, Kisliuk A, Novikov V N and Sokolov A P 2002 J. Chem. Phys. 11410189

Christensen T and Olsen N B 1994 J. Non-Cryst. Solids 172-174 357

Christensen T and Olsen N B 1995 Rev. Sci. Instrum. 665019

Doliwa B and Heuer A 2002 Energy barriers and activated dynamics in a supercooled Lennard-Jones liquid preprint cond-mat/0209139

Donth E, Beiner M, Reissig S, Korus J, Garwe F, Vieweg S, Kahle S, Hempel E and Schröter K 1996 Macromolecules 296589

Dyre J C 1991 J. Non-Cryst. Solids 135219

Ediger M D, Angell C A and Nagel S R 1996 J. Phys. Chem. 10013200

Ferry D J 1980 Viscoelastic properties of polymers (New York: 3rd ed., John Wiley)

Frick B, Zorn R, Richter D and Farago B 1991 J. Non-Cryst. Solids 131169

Gilroy K S and Phillips W A 1981 Phil. Mag. B 43735

Franosch T, Götze W, Mayr M R and Singh A P 1998 J. Noncryst. Solids 235-237 71

Götze W and Sjögren L 1992 Rep. Prog. Phys. 55241

Götze W 1999 J. Phys: Condens. Matter 11 A1-A45

Goldstein M 1968 J. Chem. Phys. 51, 3728

Hansen C, Stickel F, Berger T, Richert R and Fischer E. W. 1997 J. Chem. Phys. 1071086

Heuer A and Silbey R J 1996 Phys. Rev. B 53609

Jäckle J 1986 Rep. Prog. Phys. 49171

Johari G P and Goldstein M 1970 J. Chem. Phys. 532372

Johari G P and Goldstein M 1971 J. Chem. Phys. 554245

Johari G P 1986 Phys. Rev. B 337201

Kammerer S, Kob W and Schilling R 1998a Phys. Rev. E 582131

Kammerer S, Kob W and Schilling R 1998b Phys. Rev. E 582141

Knaak W, Mezei F and Farago B 1988 Europhys. Lett. 7529 
Kob W and Andersen H C 1995a Phys. Rev. E 514626

Kob W and Andersen H C 1995b Phys. Rev. E 524134

Kudlik A, Benkhof S, Blochowicz T, Tschirwitz C and Rössler E 1999 J.Molec.Structure 479201

Li G, Du W M, Chen X K, Cummins H Z and Tao N 1992 Phys. Rev. A 453867

Mézard M and Parisi G 1996 J. Phys. A29 6515

Mézard M and Parisi G 1999 J. Chem. Phys. 1111076

Nauroth M and Kob W 1997 Phys. Rev. E 55657

Ngai K L 2000 J. Non-Cryst. Solids 2757

Parshin D. A. 1994 Phys. Solid State 36991

Phillips W A 1981 (ed.), Amorphous Solids: Low temperature properties, (Berlin: Springer)

Pollak M and Pike G E 1972 Phys. Rev. Lett. 281449

Pusey P N and van Megen W 1986 Nature 320340

Richert R. 2002 J. Phys.: Condens. Matter 14 R703-R738

Schober H R and Oligschleger C 1996 Phys. Rev. B 5311469

Schrøder T B, Sastry S, Dyre J C and Glotzer S C 2000 J. Chem. Phys. 1129834

Sokolov A P 1998 J. Non-Crystalline Solids 235-237 190

Sokolov A P, Novikov V N, Kisliuk A and Ngai K L 2001 Phys. Rev. B 63172204

Stickel F, Fischer E. W. and Richert R 1995 J. Chem. Phys. 1026251

Stickel F, Fischer E. W. and Richert R 1996 J. Chem. Phys. 1042043

Stillinger F H 1995 Science $\mathbf{2 6 7} 1935$

Surovtsev N V, Wiedersich J, Novikov V N, Rössler E and Sokolov A P 1998 Phys. Rev. B 5814888

Taborek P, Kleiman R N and Bishop D J 1986 Phys. Rev. B 341835

van Megen W and Underwood S M 1994 Phys. Rev. E 494206

Wiedersich J, Surovtsev N V and Rössler E 2000a J. Chem. Phys. 1131143

Wiedersich J, Adichtchev S V and Rössler E 2000b Phys. Rev. Lett. 842718

Wuttke J, Kiebel M, Bartsch E, Fujara F, Petry W and Sillescu H 1993 Z. Phys. B: Cond. Matt. 91 357 